\title{
STUDIES ON HEART LYMPH*
}

\author{
BY
}

\author{
N. H. ARESKOG, G. ARTURSON, G. GROTTE and G. WALLENIUS \\ From the Departments of Paediatric and Plastic Surgery, Clinical Physiology \\ and Clinical Chemistry, University of Uppsala, Sweden
}

For some time we have been interested in problems of surgical shock and the maintenance of an adequate circulating blood volume. The ultimate aim was to find out some of the characteristics of the 'ideal' plasma-expander molecule. This led us to study the permeability of the capillary membrane, the production of lymph, the return of the proteins from the extravascular space to the circulating blood and related problems.

In the earlier studies lymph from the leg, the liver and the cervical region was collected, and the transport of various molecular sizes of dextran from plasma to lymph from these regions was measured (Grotte, 1956; Arturson, 1961). During these studies we found that lymph from the heart could be successfully collected. Lymph flows in considerable quantities from this organ and the flow is quite steady for several hours. In some experiments we also used the classical Starling heart-lung preparation (Areskog, 1962) on a dog in which we had cannulated one of the lymphatic trunks coming from the heart. As test substance, we used dextran fractions of suitable molecular distributions, with molecular weights ranging from 5,200-350,000 and also serum albumin tagged with ${ }^{131}$ I or ${ }^{125}$ I.

\section{Transport of Dextran of Molecular Weight 5,200 from Plasma to Heart Lymph}

Heart lymph was collected from a dog and the concentrations of the test substance were followed in plasma and heart lymph after a single injection of the substance (Fig. 1). It was found that the concentration of dextran in heart lymph rose rapidly and equilibrated with that of the plasma. In the latter part of the experiment the concentration of dextran in heart lymph was somewhat higher than that of plasma due to the rapid elimination from plasma via the kidneys. The dextran fraction used had a fairly 'sharp' distribution around an average molecular weight of 5,200. This corresponds to an effective

* A paper read at a meeting of the British Association of Paediatric Surgeons in Sheffield, July 1963. diffusion radius (calculated according to the formula of Einstein-Stokes) of $15 \AA\left(1 \AA=10^{-8} \mathrm{~cm}\right.$.) or the approximate size of inulin.

\section{Transport of Dextran of Molecular Weights 10,000-90,000 from Plasma to Heart Lymph}

In these experiments the transport from plasma to lymph of a dextran fraction of somewhat larger molecular weight $(10,000-90,000)$ was studied. Here the heart lymphatic was cannulated, and both kidney pedicles clamped to prevent kidney elimination of the test substance. Then dextran was introduced into the plasma by a single injection, after which the concentration of the test substance was followed in plasma and heart lymph for several hours (Fig. 2). In this case the concentration of the test substance in heart lymph soon approached that of the plasma.

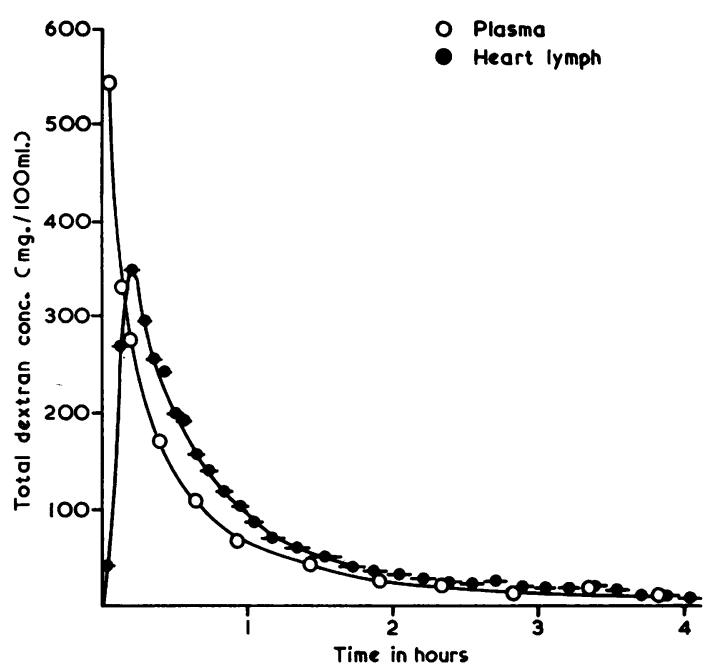

Fig. 1.-The total dextran concentration in plasma and heart lymph after intravenous injection of a 'sharp' fraction of dextran (molecular weight 5,200$) 6 \%$ in saline, $10 \mathrm{ml} . / \mathrm{kg}$. body weight in a dog. 
There is no equilibration, but after approximately two and a half hours the concentration ratio of lymph to plasma $\left(C_{\mathrm{L}} / \mathrm{C}_{\mathrm{P}}\right)$ reaches a more or less 'steady state'. These curves, however, show the total concentration of all molecular sizes $(\mathrm{M}=$ $10,000-90,000$ ) of dextran molecules in plasma and lymph respectively. When the 'steady state' is reached after about two and a half to three hours, the distribution of the various molecular weights is determined according to the method of Wallenius (1954) and Wallenius, Waschewsky, Aronsson and Hegedüs (1960). The concentration of each molecular weight class of dextran in lymph can then be compared with that of plasma, as shown in Fig. 3, where the ratio $C_{\mathrm{L}} / \mathrm{C}_{\mathrm{P}}$ is plotted against the molecular size of the test substance. This figure demonstrates the relative obstruction presented by the capillary wall to various sizes of dextran molecules. The dextran molecule with $M$ about 5,000 is obviously rapidly equilibrated, i.e. the ratio $C_{L} / C_{P}$ is unity (Fig. 1), but for molecular weights of about 30,00040,000 the ratio $C_{\mathrm{t}} / \mathrm{C}_{\mathrm{P}}$ falls rapidly.

These results are in good agreement with earlier experiments, where lymph was collected from the leg and the cervical region of the dog. Accepting the pore theory of capillary permeability, we believe that these water-soluble molecules pass through some aqueous channels probably situated between the endothelial cells of the capillary wall. The size of these pores must then be somewhat larger than the passing molecules and, from the theories of Pappenheimer (1953), it may be calculated that, if the membrane is fairly isoporous, the radius of these pores must be about 35-45 $\AA$ (Arturson, Areskog, Grotte and Wallenius, to be published).

\section{Transport of Dextran of Molecular Weight 100,000-350,000 from Plasma to Heart Lymph}

Already in 1931 Drinker and Field demonstrated the normal presence in lymph of all the plasma proteins and the extravascular circulation of the plasma proteins.

After injection of dextran of larger molecular weights $(100,000-350,000)$, all these molecular sizes are found in heart lymph in about the same proportions as in the plasma, although the total concentration in the lymph is much lower than that in the plasma. Fig. 4 shows that the ratio $C_{L} / C_{P}$ for these larger molecules is fairly constant with increasing molecular size, i.e. the transport of these larger molecules from plasma to heart lymph seems quite independent of molecular size. The effective diffusion radii of these larger dextran molecules have a magnitude of about $120 \AA$. Their presence in lymph can then only be explained by the

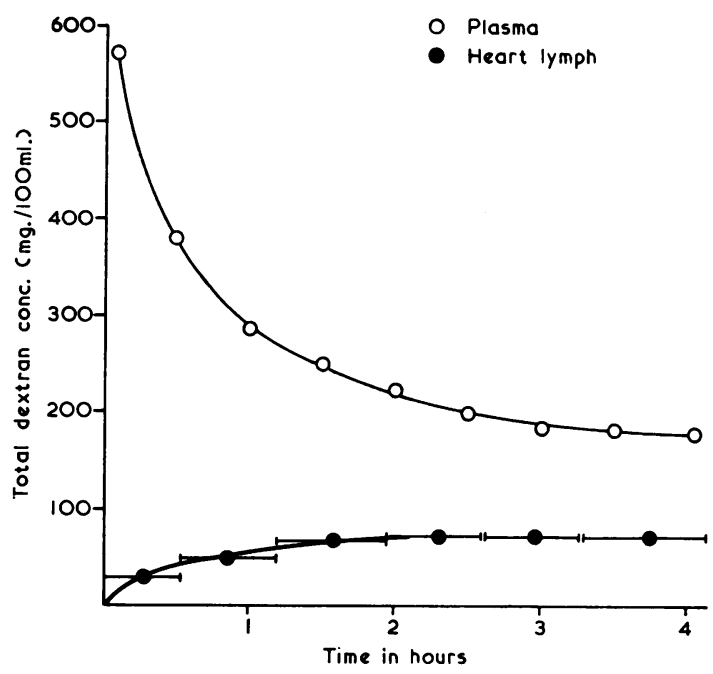

Fig. 2.-The total dextran concentration in plasma and heart lymph after intravenous injection of dextran with molecular weight 10,000 $90,000,6 \%$ in saline, $10 \mathrm{ml} . / \mathrm{kg}$. body weight in a dog with occluded renal circulation.

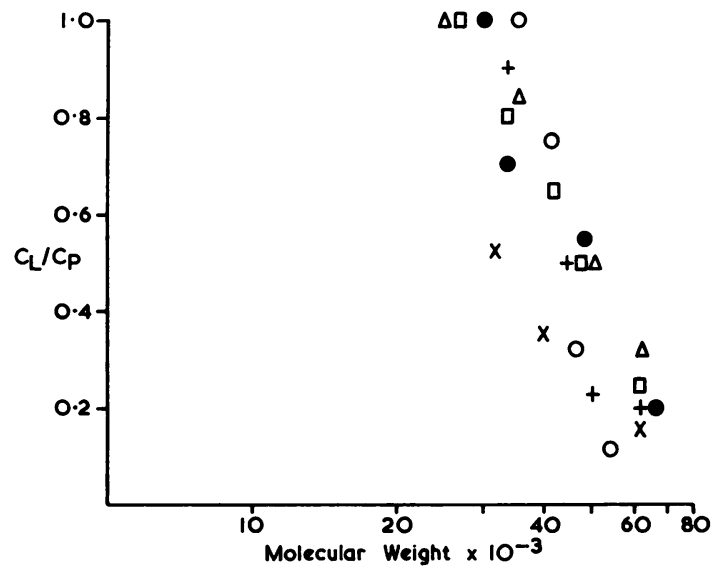

FIG. 3.-The lymph/plasma concentration ratio $\left(C_{L} / C_{P}\right)$ plotted against molecular weight after intravenous injection of dextran with molecular weight $10,000-90,000,6 \%$ in saline, $10 \mathrm{ml} . / \mathrm{kg}$. body weight in four heart-lung preparations $(x, \Delta,+, \square)$ and in two dogs with occluded renal circulation $(\mathrm{O}, \mathrm{O})$

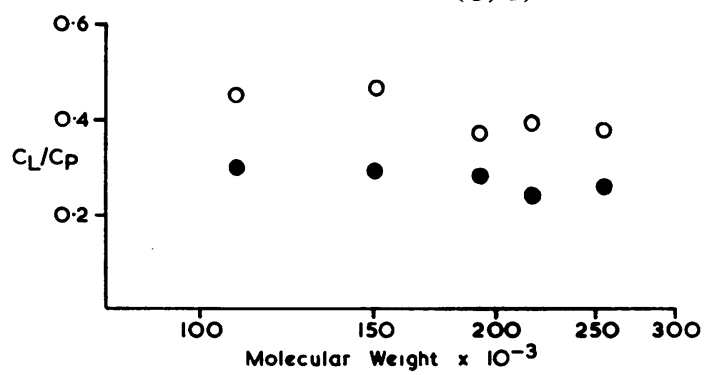

FIG. 4.-The lymph/plasma concentration ratio $\left(C_{L} / C_{P}\right)$ plotted against molecular weight after intravenous injection of dextran with molecular weight $100,000-350,000,6 \%$ in saline, $10 \mathrm{ml} . / \mathrm{kg}$. body weight in two dogs not occluded $(O, O)$. 
presence in the capillary membrane of larger 'leaks' of at least this dimension. Since the concentration of these larger molecules is usually low but of a fairly constant value, we must assume a relatively constant frequency of these 'leaks' under normal conditions. Similar 'leaks' have been demonstrated in the leg and cervical region. In the heart experiments a higher $\mathrm{C}_{\mathrm{L}} / \mathrm{C}_{\mathrm{P}}$ ratio for these larger molecules was found. This may be explained by a larger number of 'leaks' in the heart capillaries. Using plastic balls (methylmethacrylate) with an effective radius of about 300$700 \AA$, we have shown that these balls, which have approximately twice the size of the larger plasma proteins, do not pass from plasma to heart lymph. This indicates that the radius of these capillary 'leaks' is about $120-300 \AA$ (Grotte, Juhlin and Sandberg, 1960).

\section{Transport of ${ }^{131}$ I Albumin From Plasma to Heart Lymph}

In order to study the transport of protein molecules from plasma to lymph, serum albumin tagged with ${ }^{131}$ I and ${ }^{125}$ I (human as well as dog serum albumin) was used as a test substance. In these experiments dog heart-lung preparations were used, in which the lymphatic draining mainly the left side of the heart was cannulated. After the operative procedures, when the preparation was working steadily, the test substance was injected into the circulating blood. The concentration of this test substance was followed in plasma and heart lymph for several hours. A typical experiment is illustrated in Fig. 5. In the heart-lung preparation the total capillary surface area is considerably reduced compared with that of the whole animal, and the plasma concentration of the tagged serum albumin is thus kept fairly constant for several hours.

After injection of the test substance the concentration in heart lymph rises rapidly, approaching that of the plasma. The concentration increase of the labelled substance in heart lymph shows a time course indicating that from the intravascular compartment the substance was mainly distributed to a single extravascular compartment. The data could thus be explained by a two-compartment model and the permeability constant and the size of the extravascular albumin space drained by the lymphatic cannula could be calculated (Grotte, Areskog and Arturson, to be published).

Theoretical values for the rise of the concentration of ${ }^{131}$ I albumin in heart lymph were calculated according to the equation

$$
\mathrm{C}^{*}{ }_{\mathrm{L}}=\frac{K_{1}}{V_{E} \cdot K_{2}} A^{*}{ }_{P}\left(1-\mathrm{e}^{-K_{2} t}\right)
$$

(Solomon, 1953), where $\mathrm{C}^{*} \mathrm{~L}=$ concentration of ${ }^{131} \mathbf{I}$

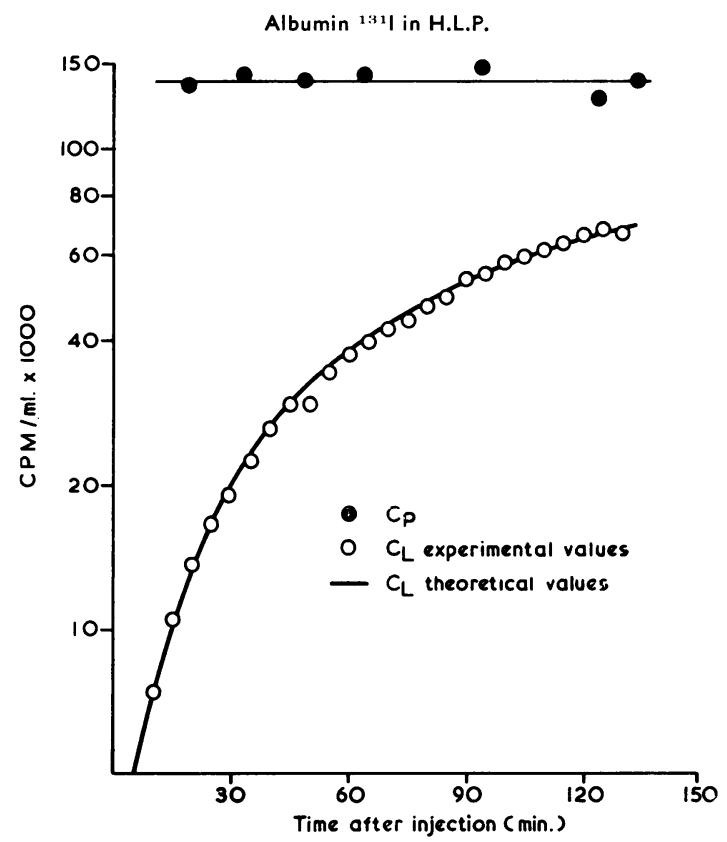

FIG. 5.-The activity of ${ }^{131} \mathrm{I}$ albumin in plasma and heart lymph in a heart-lung preparation. Both the theoretical values for the rise of the activity of ${ }^{131} \mathrm{I}$ albumin in heart lymph and the experimental values are plotted against time after injection.

albumin in heart lymph, $V_{E}=$ volume of extravascular distribution of tracer, $K_{1}=$ constant of transfer of tracer from plasma to extravascular compartment, $K_{2}=$ constant of transfer from the extravascular compartment to outside (lymph cannula). $A^{*}{ }_{P}=$ amount of ${ }^{131} \mathbf{I}$ albumin in plasma, and $t=$ time.

As shown in Fig. 5, the experimental values are in good agreement with those valid for a two-compartment model. It can be shown that the extravascular distribution volume of the tracer $\left(V_{E}\right)$ may be calculated from the formula $V_{E}=\frac{L}{K_{2}}$, where $L=$ lymph flow and $K_{2}=$ same as above. In one out of seven successful experiments the values determined were the following:

Heart weight $=88 \mathrm{~g} . \quad V_{E}=6.1 \mathrm{ml} . \quad K_{1}=0.075 /$ $\min . \quad K_{2}=0.0067 / \mathrm{min}$.

\section{Transport of Albumin From Extravascular Space back to Circulating Blood}

In 1931 Drinker and Field stated that all the blood capillaries leak protein and that this protein is returned to the circulating blood via the lymphatics. Since that time it has been generally assumed that all proteins that have leaked out through capillary walls are returned via the lymphatics and that there is no direct return of proteins from the extravascular space back to the blood through the capillary walls. The validity of this assumption has been questioned many times, and experimental evidence for the more 


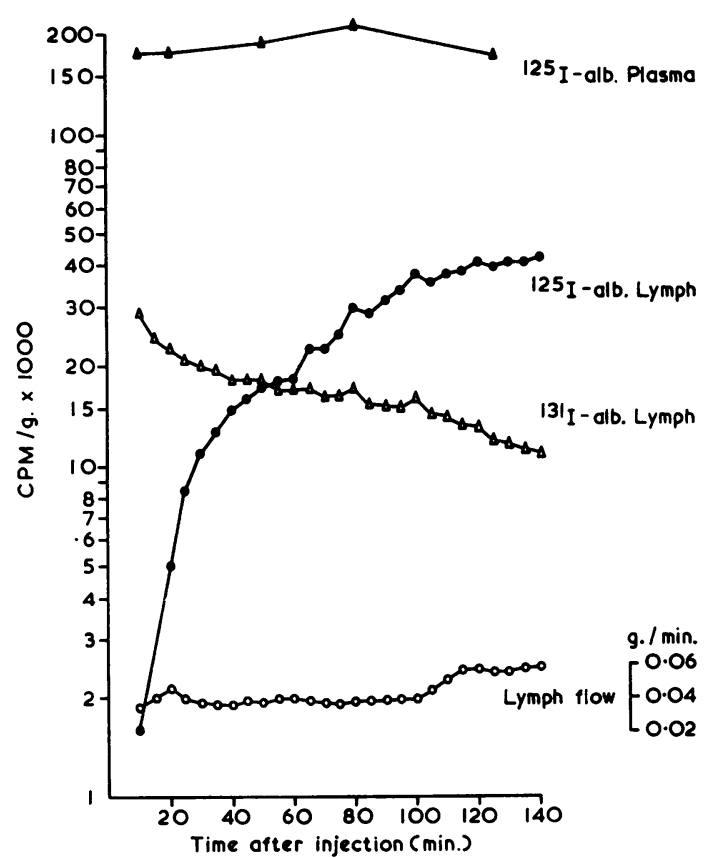

Fig. 6. - The activities of ${ }^{131} \mathrm{I}$ albumin and ${ }^{125} \mathrm{I}$ albumin in plasma and heart lymph and the lymph flow in a heart-lung preparation plotted against time after an exchange transfusion, whereby all the circulating blood containing ${ }^{131}$ I albumin previously injected was replaced by blood containing ${ }^{125}$ I albumin.

direct transport has recently been given by Swann, Stegall, Collings and Miles (1961).

We have tried to elucidate this problem in the following way. A tracer dose of ${ }^{131}$ I albumin was injected intravenously into a dog, and 24 hours later, when the tracer had reached a steady concentration in the heart lymph, the heart-lung preparation was prepared and the lymphatic from the heart was cannulated. As soon as the condition of this preparation was satisfactory and steady, an exchange transfusion was made, whereby all the circulating blood containing ${ }^{131} \mathrm{I}$ albumin was replaced by blood containing ${ }^{125} \mathrm{I}$ albumin in a tracer dose. The concentration of ${ }^{125} \mathrm{I}$ albumin was then steady in the plasma for several hours. The rise of the concentration of ${ }^{125} \mathrm{I}$ albumin was followed in the heart lymph, as well as the fall of the concentration of the now only extravascularly distributed ${ }^{131}$ I albumin (Fig. 6). With the aid of ${ }^{125}$ I albumin the extravascular distribution volume of albumin in this preparation could be calculated from the equations given above. When both the extravascular distribution volume and the fall in the activity of ${ }^{131} \mathrm{I}$ albumin are known, it can be ascertained whether all the ${ }^{131}$ I albumin appears in the lymphatic cannula or if there are any other transport routes, e.g. directly through the capillary wall back to the circulating blood. Our preliminary data show that the direct transport of albumin from the extravascular compartment back to the circulating blood is of minor importance and that the main transport route is via the lymphatics.

\section{Summary}

The sieving characteristics of the capillary membranes of the dog's heart were determined by using dextran molecules of different sizes. The results were interpreted according to the pore theory of capillary permeability, i.e. water-soluble molecules pass through 'aqueous channels' between the endothelial cells. It seems possible that the capillary regions so far investigated by this method (leg, cervical regions and heart) have a similar functional ultra-structure: the capillary membranes are penetrated by a large number of pores of an approximate radius of $35-45 \AA$ and also by 'leaks' of a radius of about $120-300 \AA$ but few in number. These results are to some extent in agreement with investigations using electron microscopic techniques (Bennett, Luft and Hampton, 1959).

When using dextran as a plasma expander our studies corroborate the view that dextran of molecular weight of about 50,000 , i.e. slightly above the kidney threshold, will be adequate for volume expansion, as judged during normal physiological conditions. This is also supported by studies of plasma disappearance of dextran in humans (Arturson and Wallenius, 1964). The effective diffusion radius of these dextran molecules is similar to that of serum albumin. Our studies have also given some quantitative data on the transport kinetics of albumin in the heart tissue of the dog, its volume of extravascular distribution and the recirculation of albumin from the extravascular compartment.

This study was supported by generous grants from the Swedish National Association against Heart and Chest Diseases, Stockholm, the Swedish Medical Research Council and AB Pharmacia, Uppsala, Sweden.

We wish to express our sincere gratitude to our laboratory engineer, Mr. Joachim Waschewsky, for excellent technical aid, especially concerning the molecular-weight determination of dextran.

\section{REFERENCES}

Areskog, N. H. (1962). Experimental Studies on Myocardial Function. Acta Universitatis Upsaliensis, Almqvist and Wiksell, Uppsala. Arturson, G. (1961). Pathophysiological Aspects of the Burn Syndrome. Acta chir. scand., Suppl. 274.

Areskog, N. H., Grotte, G. and Wallenius, G. Studies on heart lymph. II. Capillary permeability of the dog's heart. To be published.

and Wallenius, G. (1964). The intravascular persistence of dextran of different molecular sizes in normal humans. Scand. $J$. clin. Lab. Invest. In the press.

Bennett, H. S., Luft, J. H. and Hampton, J. C. (1959). Morphological classifications of vertebrate blood capillaries. Amer. J. Physiol., 196, 381 . 
Drinker, C. K. and Field, M. E. (1931). The protein content of mammalian lymph and the relation of lymph to tissue fluid. ibid., 97, 32.

Grotte, G. (1956). Passage of dextran molecules across the bloodlymph barrier. Acta chir. scand., Suppl. 211.

Areskog, N. H. and Arturson, G. Studies on heart lymph. I Kinetics of $I^{131}$ albumin in the dog heart-lung preparation. To be published.

-..-, Juhlin, L. and Sandberg, N. (1960). Passage of solid spherical particles across the blood-lymph barrier. Acta physiol. scand. 50, 287.

Pappenheimer, J. R. (1953). Passage of molecules through capillary walls. Physiol. Rev., 33, 387.
Solomon, A. K. (1953). The kinetics of biological processes. Special problems connected with the use of tracers. Advanc. Biol. Med. Phys., 3, 65.

Swann, H. G., Stegall, H. F., Collings, W. D. and Miles, N. A. (1961). Red cell and albumin circulations in the ileum. Amer.J. Physiol., 201, 943.

Wallenius, G. (1954). Renal Clearance of Dextran as a Measure of Glomerular Permeability. Acta Soc. Med upsalien., Suppl. 4. Waschewsky, H. J., Aronsson, T. and Hegedüs, L. (1960) Automatic Turbidimeter for Determination of Molecular Weight Distribution of Polymers. Proc. of Fifth int. Instrum. Measurem. Conf., Stockholm, Sweden. 